\title{
Novel Robotic Assistive Technologies: Choosing Appropriate Training for Healthcare Professionals
} \author{
$\operatorname{Ragot}^{5}$, P. Gallien ${ }^{3}$ \\ ${ }^{1}$ CHU Rouen, Institute of Formation in Ergotherapy, Rouen F-76000, France \\ ${ }^{2}$ Breizh Cerebral Palsy Network, Rennes, France \\ ${ }^{3}$ Saint Hélier Pole, Rennes 35000, France \\ ${ }^{4}$ Canterbury Christ Church University, UK \\ ${ }^{5}$ UNI-Rouen, Normandy University ESIGELEC, Rouen, France
}

L. Ménard ${ }^{1}$, A. Petit ${ }^{2}$, É. Leblong ${ }^{3}$, M. Stein ${ }^{4}$, E. Hatzidimitriadou ${ }^{4}$, R. Khemmar ${ }^{5}$, S. Manship ${ }^{4}$, R. Morris ${ }^{4}$, N.

Corresponding Author Email: lucie.menard@chu-rouen.fr, axel.petit@pole-sthelier.com, emilie.leblong@pole-sthelier.com, maria.stein@canterbury.ac.uk ; eleni.hatzidimitriadou@canterbury.ac.uk, redouane.khemmar@esigelec.fr, sharon.manship@canterbury.ac.uk; rachael.morris@canterbury.ac.uk,nicolas.ragot@esigelec.fr,philippe.gallien@polesthelier.com

https://doi.org/10.18280/mmc_c.811-408

Received: 30 June 2020

Accepted: 18 October 2020

\section{Keywords:}

assistive technologies, training, disability, ADAPT European project

\begin{abstract}
One of the key challenges for the training of healthcare professionals (HCPs) is to maintain a good understanding and knowledge of new assistive technologies (ATs) that are currently on the market [1]. Indeed, at present, available training on ATs is limited and does not meet the practice-related needs of HCPs. It is in this context that the ADAPT European project aims to develop a new AT training programme for healthcare professionals, which will also introduce them to the project's new AT developments - a smart powered wheelchair and a virtual reality wheelchair-driving simulator. The program consists of six multimodal units; five delivered via e-learning and one through a blended method of e-learning and face-to-face sessions. The development of the content is guided by findings from an earlier literature review and an online survey of AT training needs for HCP's, both undertaken by the ADAPT cross-national research team, comprised of UK and French experts. The level of technical difficulty increases with successive units in order to train all HCPs to use innovative ATs more widely in their practice. A Learning Management System enables the dissemination of the e-learning AT program. Preliminary results from participant unit-specific evaluations available at this stage are overall positive and encouraging.
\end{abstract}

\section{INTRODUCTION}

The introduction of novel Assistive Technologies (ATs) aimed at helping people with disabilities has highlighted the need to enhance education and training of healthcare professionals (HCPs) in their use and application.

However, how best to optimize AT training for HCPs is not very clear due to a variety of reasons. For example, the extent to which novel technologies modify prescribing habits is still to be determined. In addition, challenges already exist around the use and acceptance of low-tech AT in practice. This makes it more difficult to prepare HCPs for challenges associated with use of more complex high-tech AT. Internationally, the level of knowledge and skills in ATs among HCPs is not well known either. One of the aims of the ADAPT project (Assistive Devices for empowering disabled People through robotic Technologies) is to address some of the challenges associated with AT training of HCPs.

The project aims to improve the comfort and quality of life of people with reduced mobility and loss of autonomy, by using robotic assistive technologies. It is made up of a consortium of $16 \mathrm{UK}$ and French partners, funded by the EU
INTERREG VA program, and based around the development of:

- A smart and connected powered wheelchair (SPW), incorporating a driving assistance in order to enhance the user's autonomy.

- A virtual reality wheelchair-driving simulator, for an immersive experience of the wheelchair.

- Development of an AT training programme intended for HCPs.

- A partnership between academic research institutions and companies, in order to work collaboratively promote the project outcomes.

The work of this paper pertains to the development of the AT training program for HCPs. The goal of this project is to train a minimum of $680 \mathrm{HCPs}$ in France and in the United Kingdom, through e-learning and face-to-face training sessions. Each partner contributed specific expertise in engineering, medical, health care and pedagogical fields in order to inform the design and content of the AT training program, which includes training in the use and application of the smart wheelchair and the virtual reality (VR) wheelchairdriving simulator. Hence, the training program is enriched by 
novel robotic assistive technologies developed in the ADAPT project; the SPW and the virtual reality simulator.

In addition to its educational value, the ADAPT training is designed to collect data on evaluation data on the learning from the training of HCPs. These data will be used to monitor and further enhance the development of training and for comparison purposes in uptake of and learning from the training amongst French and UK healthcare professionals.

\section{STATE OF THE ART}

\subsection{Education offer and training needs in assistive technology for healthcare professionals}

ATs can be efficient and correctly applied in healthcare practice only if particular attention is paid to their setting up, which has to be adapted, explained and supported [2]. Therefore, training HCPs to these issues appears to be crucial.

In France, there is AT education for HCPs which is deployed as part of pre-registration or continuing professional development training. There are several University Diplomas that relate to ATs and orthopedic devices, as well as vocational training such as that offered by the French National Association of Occupational Therapists (ANFE) or by the French Association against Myopathies (AFM). Allied Health Schools also train future occupational therapists, physiotherapists or speech and language therapists to this topic.

Studies included in the ADAPT literature review [3] show that HCPs have increasing training needs for understanding and knowledge about available AT devices and systems as well as current research on robotic assistance [4, 5]. More specifically, the National Observatory on Formation, Research and Innovation on Handicap (ONFRIH) has made a number of recommendations in France relating to the points AT training should focus on. In particular, it is suggested that AT training should deal with improving prescription, acquiring and adapting novel self-help devices. The ONFRIH identifies three target audiences: prescribers, equipment suppliers, and users and their careers [6]. Other studies insist on the fact that the disciplines dealing with AT should be taught more in depth during their pre-registration Higher Education studies, and therefore AT training should be incorporated at that level [712]. Hence, it can be challenging to develop good quality training to address the diverse training needs of all these target audiences [13]. Furthermore, it has been highlighted that a combination of theoretical and practical content in AT would promote the acquisition of practice-related skills [14].

\subsection{Multimodal training: Key issues}

While Biard shows that practice education is more efficient when delivered face-to-face rather than online, he also notes that online training reduces the cognitive load due to the segmentation of learning sequences and breaks. In addition, the possibility for learners to control the access to information improves the memorization of theoretical concepts. Face-toface training is more relevant for developing specific practice skills [15].

Based on a thorough literature review, the U.S. Department of Education highlights several quality criteria to ensure memorization and satisfaction with online learning [16]:

- Regular interventions by the trainer
- Collaborative work between learners and between learners and trainer

- $\quad$ Physical meeting between learners and trainer during the programme (multimodal training)

- $\quad$ Exchanges with the trainer and other learners during the training

- Use of non-text media (photo, videos, oral messages)

- Individualised instructions

- $\quad$ Practice during training

- $\quad$ Flexibility of online learning time

- $\quad$ Self-checking and self-reflection exercises

- Additional resources in the event of an incorrect answer to a quiz question

Multimodality is a crucial element of effective education. Combining a face-to-face session with the provision of online learning resources allows, among other things, the repetition of key messages, freedom of consultation and access to various means of communication with trainers and between learners [16].

Furthermore, online training has an additional advantage. Information and communication technologies offer opportunities for wide dissemination and avoid the logistical constraints that would have made face-to-face delivery impractical, especially in cases where learners work and reside in different regions and countries, as with the ADAPT AT training program.

\section{OUR PEDAGOGICAL APPROACH: MULTIMODAL AND INCREMENTAL AT TRAINING}

\subsection{Analysis of training needs}

Developing AT training for HCPs poses significant challenges. Although HCPs have a rich practical and theoretical knowledge base, consideration must be given to the fact that up to date, the bulk of their work revolves around social interactions and manual techniques.

In order to develop innovative, adapted and relevant training, we had to analyze more closely the needs of HCPs in terms of new ATs training. To do this, the project partners carried out a scoping literature review, as outlined in section II. A. Findings from the review highlighted certain gaps in AT training for HCPs $[17,18]$. To explore these gaps further and inform the development of the ADAPT training, an online survey was undertaken in both countries in order to identify the knowledge, experience and training needs of HCPs in the field of TA $[19,20]$. The survey consisted of 37 semi-openended questions, it was completed by 368 participants in France and the United Kingdom. Survey results highlighted the following points.

According to survey participants, current provision of vocational AT training and training for AT users was limited. Moreover, in the United Kingdom, such training was not readily available and it was considered inadequate by $76.6 \%$ of survey participants. In France, the majority of HCPs made the same observation for continuing education (46.7\%), while pre-registration AT training was considered to be quite sufficient by $72.8 \%$ of French survey respondents.

The following areas were identified as key needs for AT training: overall knowledge about ATs and knowledge about specific AT systems; prescription of ATs; follow-up and 
abandonment of ATs; user assessment; AT user assessment; AT user training; and customized adaptation of ATs.

In addition, survey findings indicated that HCPs were interested in knowing more about evidence-based AT practice for different AT user groups. Also, the need for availability of online training readily accessible in various workplaces such as hospitals with rehabilitation departments was highlighted by the survey.

\subsection{Training program}

The needs identified in the ADAPT literature review and the online survey informed the content of the ADAPT AT training program. This is based on six learning units of increasing technical difficulty. The first five units will be delivered via elearning and the last one is delivered through a blended approach of e-learning and face-to-face sessions. The program begins with Foundations of ATs and Understanding Disability. It concludes with the prospects offered by the novel robotic assistive technologies developed in the ADAPT project. This training program aims to contribute to the development of AT knowledge and skills among learners, two essential components for professional practice.

The target groups for the delivery of the training program are healthcare professionals and students. However, users and caregivers would be also able to undertake some or all of the training units depending on their interest and particular training needs. The first two units are aimed at professionals interested in knowing more about AT and how it can increase independent living. Units 3 to 6 are more suitable for experienced professionals who already have some knowledge of AT but would like to increase the adoption of AT solutions in their practice.

Unit 1 and 2 are awareness raising units about ATs and disability. Both are structured around pedagogical objectives such as: knowing essential definitions, understanding models of disability, and classifying ATs according to their technology level or their categories (such as mobility, communication or cognitive functions). Both units are more theoretical than the rest of the program. This ensures consistency amongst learners that they have a sound basis for understanding the rest of the units. Indeed, when considering AT solutions, it is very important to understand the person's disability as a whole in order to prescribe the most appropriate AT devices and systems. This increased knowledge and awareness was one of the identified needs by HCPs in the literature review (see section 2.1).

Unit 1 is a general AT unit. It gives the definition of ATs, presents their different technological levels (low, mid and high tech) as well as examples of the AT use depending on the category to which they belong (AT for mobility, cognitive functions or communication for example). Unit 1 also presents policies and regulations governing acquisition of $\mathrm{AT}$, funding sources and rights and duties of an AT user. Unit 2 deals with the understanding of disability in a broader sense. In particular, it allows learners to appreciate different definitions and models of disability including the medical, social and biopsycho-social model. The last model provides the basis of the International Classification of Functioning (ICF) (WHO 2001), which is of important relevance to AT prescription and solutions. Unit 2 also highlights differences in French and British law in regard to management of disability and AT service provision. In summary, learners become more aware of the importance of understanding disability for the use of AT in Health and Social Care. Apart from raising awareness and providing knowledge about new generations of AT, ADAPT training seeks to develop professional skills for the use of new assistive technologies. In order to provide professionals with tools to advance their practice and enable the transfer of learning into their daily work, we have focused the learning objectives of units 3 to 6 towards the acquisition of professional knowledge and skills relating to ATs.

The focus of Units 3 and 4 is specific areas of AT applications - ATs for mobility and posture and ATs for communication respectively. The training in Unit 3 forms a sound basis for the presentation of and training in novel robotic assistive technology for wheelchair users which is developed in the ADAPT project. Furthermore, the importance of communication ATs, which is presented in Unit 4, is an issue highlighted by the UK ADAPT Advisory Group, which impacts on people with mobility disabilities who are likely to use the smart wheelchair.

Definitions of key aspects for understanding ATs for posture and mobility and communication are also presented in the first part of Units 3 and 4, followed by three case studies in the second part. They highlight, in a concrete way, how people affected by mobility and/or posture and communication disorders. Then, they focus on practical issues: user assessment, prescription of the most appropriate AT and the benefits of AT in daily life. Here again, these themes meet the needs identified by HCPs in the ADAPT online survey (see 3.1). At the end of these two units, learners are able to use the professional vocabulary adapted for these fields, to understand the holistic assessment of the AT user and to recommend ATs that are customized taking into account the individual's needs and situation.

Unit 5 addresses one of the needs identified by HCPs i.e., appraising the evidence underpinning use of AT so that they apply evidence-based practice (EBP). More than just providing the learner with a list of evidence for a particular AT solution, the training imparts key research skills necessary for undertaking their own research in this area. For example, methodologies for relevant literature searches, critical analysis and synthesis of evidence provide a sound basis for the application of recommended ATs. By the end of this Unit, the learners are able to understand what EBP is as well as its importance in guiding and informing AT research. They will be able to analyze AT-related literature using appropriate assessment tools, to determine the validity and relevance of the evidence, and finally to apply EBP to their practice in order to make it evolve.

The sixth and final Unit is focused on the novel technologies developed in the ADAPT project: the smart wheelchair and the virtual reality wheelchair-driving simulator. The aim is to prepare HCPs for the launch of high-tech ATs on the market and to support their uptake in future practice.

Unit 6 is thus part of the continuous improvement of patient care and contributes to increasing the development and adoption of innovative products, processes, systems and services in the medical and allied health fields. It enables professionals to acquire the necessary skills to use these technologies and, above all, to reuse them in their practice with their patients in order to enrich their care, autonomy and independent living. In addition to the existing data in the scientific literature, the content of Unit 6 is based on the experiments and clinical trials specific to the ADAPT project [21]. These clinical trials allow objective assessment of the ATs' relevance and highlight areas for improvement in the 
development and uptake of ADAPT novel technologies. At the end of this Unit, participants are able to handle and use the smart wheelchair in order to recommend it as AT for independent mobility. Training will also be provided on the specific wheelchair-driving simulator and its prototypes.

Therefore, the ADAPT AT training as a whole is an incremental and coherent program that addresses several gaps in the literature and survey, as identified by the project team.

\subsection{Pedagogical strategy}

Nine of the ten quality criteria listed in 2.2 for open distance learning apply to the ADAPT AT training. In relation to the criterion that is not met, delivering individualized instructions, it requires a technically time-consuming setting up of variables, and the deliveries imposed by the project do not allow to carry them out. The other nine criteria are applied in various forms. The ADAPT AT learning materials and methods, as presented in the list below, meet one or more of the identified quality criteria:

- Videos from the training's referents punctuating the pedagogical content at regular intervals.

- Collaborative exercises with questions/answers on an online forum.

- One learning unit delivered in a multimodal format.

- Videos and interactive animated modules.

- Exercises to search for AT on a dedicated French platform.

- $\quad$ Free access to the modules.

- $\quad$ Self-reflection exercises.

- Remedies in case of negative answer to the quiz questions.
The design of the training by applying these learning methods helps to ensure its quality. The impact of the training will be measured using the evaluation methods described in part 4.2.

\section{RESULTS}

\subsection{Development of training materials}

The method used to develop the pedagogical content involves a variety of professionals with expertise in disability, pedagogy and robotics engineering. A multidisciplinary approach to the training is particularly important from a pedagogical point of view, given the complexity of the technology and the use of ATs [22]. For each learning unit, a storyboard is first drawn up: this is a shared working document, which structures the content and lists the training sequences, the resources used and the teaching methods to be employed. HCPs with expertise in medicine, occupational therapy and physiotherapy write the document. Once validated, an elearning professional creates interactive modules and integrates the resources using specialised tools. The entire system is then integrated into a platform dedicated to open distance learning, a Learning Management System (LMS) (Figure 1).

This LMS ensures the dissemination of training and data collection for statistical purposes. In particular, it collects data on learner attendance, time spent in training and the results of evaluation questionnaires. The system should also provide learners with a fluent, simple and clear learning experience in order to limit cognitive load and allow learners to focus efficiently on the learning content [23].

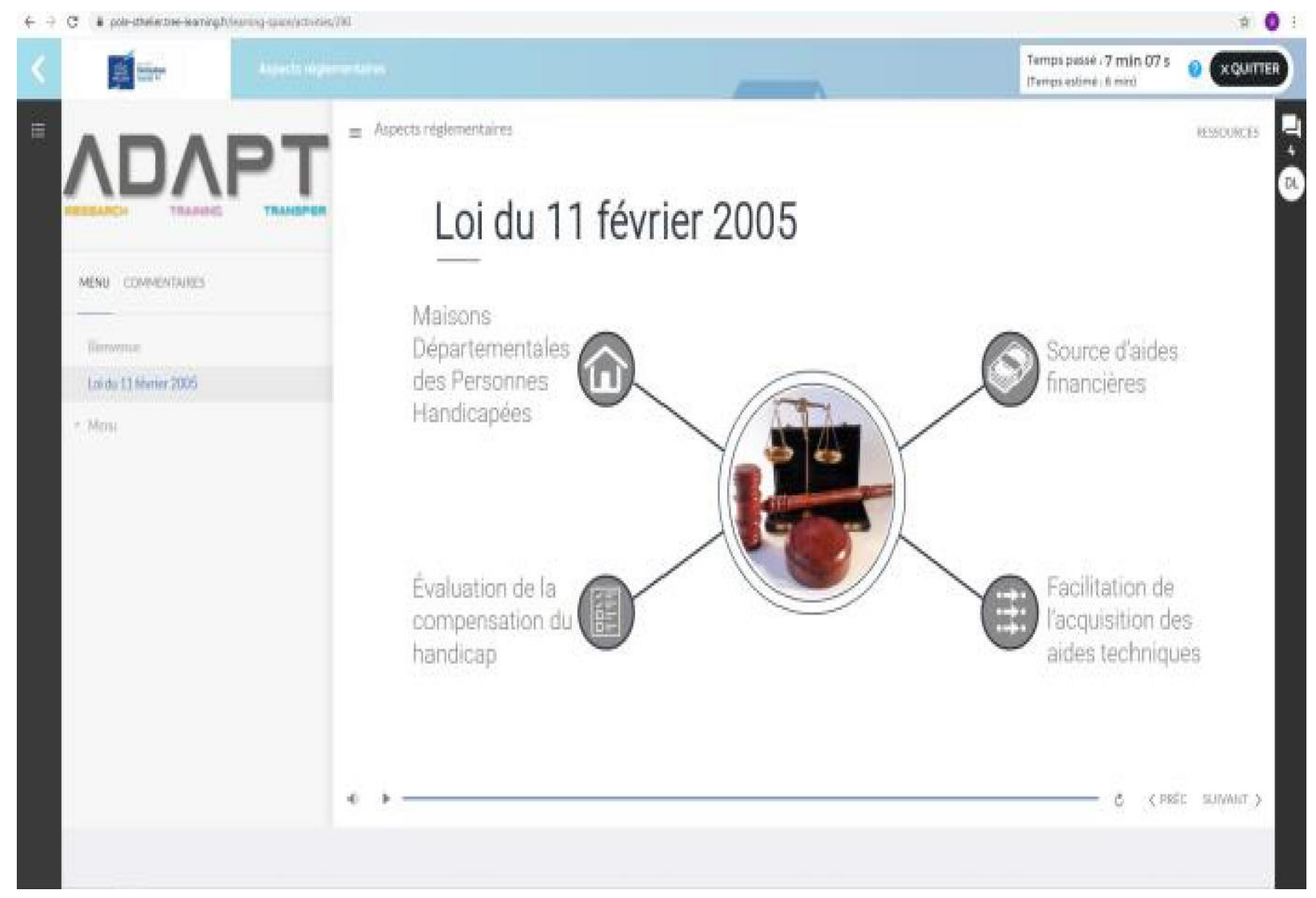

Figure 1. Example of a screen on the ADAPT LMS 


\subsection{Evaluation of learning}

The delivery of the ADAPT AT training program is being evaluated for the purposes of the project in several ways. First, we monitor the number and type of learners engaging with each Unit and the whole training program as a reporting requirement to the funder. In particular, we record the number of both healthcare students and professionals who complete each Unit in both countries. In addition, we are also collecting information about the length and level of work experience and in order to appreciate how the new training program enables newly qualified and experienced professionals to develop new AT practice skills.

Also, learners are requested to complete a short online evaluation questionnaire, developed by the ADAPT training team, at the end of each Unit and six months after the completion of the training as a follow-up. The evaluation questionnaire consists of 16 single-choice and open-ended questions. The learners are requested to rate their level of knowledge, skills and confidence using AT before and after the completion of the training units and their readiness to use AT in practice. They evaluate the relevance of the content, its adequacy with the pedagogical objectives and the needs of professionals, the layout of the content and the degree of interactivity offered by the e-learning platform. At the time of writing, evaluation data was available for Unit 1 and 2 as the remaining units are yet to be launched.

Preliminary analysis of the results from Unit 1 are available for 124 learners (the majority of them are healthcare students). Results show an increase in the ratings for knowledge, confidence and readiness to use ATs (learners increase on average from $3.5 / 5$ to $4.3 / 5$ regarding their knowledge). The majority $(95 \%)$ of the learners felt that the content met its pedagogical objectives, $93.5 \%$ felt that the content was appropriate and relevant, and almost all learners found it interesting (96\%) and easy to follow (98.4\%). Finally, $87.1 \%$ of learners perceived at least one skill that they could transfer into their practice.

The results are useful on several levels. Firstly, knowing the number of learners who undertake the training allows us to monitor the extent to which the third objective of the ADAPT project is achieved i.e., the minimum target of 680 HCPs to be trained in the UK and French regions bordering the Channel. Secondly, post-training evaluation results inform further development of the Units, ensuring that the ADAPT training program remains up to date and meets the changing needs of HCPs.

\section{CONCLUSION}

With the emergence and introduction of novel technologies in the workplace, the prescription of ATs is rapidly changing. Findings from the scoping literature review and online survey conducted by the ADAPT project team demonstrate that the training of HCPs in new assistive technology has become a necessity for their practice. Beyond high-tech ATs, it appears that a more comprehensive training concerning less advanced (low and mid tech) ATs is necessary.

By delivering multimodal training that meets widely accepted quality criteria for distance learning, the ADAPT project will contribute to the efficient training of these HCPs in France and the UK. Preliminary results of post-training evaluation are encouraging. The forthcoming delivery of the whole ADAPT AT training program will provide more data and confirm the value of our innovative education approach and impact on AT practice.

\section{ACKNOWLEDGMENT}

This work is carried out as part of the INTERREG VA FMA ADAPT project "Assistive Devices for empowering disabled People through robotic Technologies". The Interreg FCE Programme is a European Territorial Cooperation program that aims to fund high quality cooperation projects in the Channel border region between France and England. The Program is funded by the European Regional Development Fund (ERDF).

\section{REFERENCES}

[1] Association Nationale pour la Formation permanente du personnel Hospitalier. (2020). Plan d'actions régional. https://www.anfh.fr/sites/default/files/fichiers/par_2020 _nomandie_interactif.pdf

[2] Widehammar, C., Lidström, H., Hermansson, L. (2019). Environmental barriers to participation and facilitators for use of three types of assistive technology devices. Assistive Technology, 31(2): 68-76. https://doi.org/10.1080/10400435.2017.1363828

[3] Manship, S., Hatzidimitriadou, E., Stein, M., Parkin, C., Raffray, M., Gallien, P., Delestre, C. (2019). A literature review of the challenges encountered in the adoption of assistive technology (AT) and training of healthcare professionals. Association for the Advancement of Assistive Technology in Europe (AAATE), pp. 90-91. https://kar.kent.ac.uk/76435/

[4] Brose, S.W., Weber, D.J., Salatin, B.A., Grindle, G.G., Wang, H., Vazquez, J.J., Cooper, R.A. (2010). The role of assistive robotics in the lives of persons with disability. American Journal of Physical Medicine \& Rehabilitation, 89(6): 509-521.

[5] Malcolm. (2015). Assisted living capability analysis report. https://sehta.co.uk/Post/?permalink=malcolmreports

[6] ONFRIH. (2011). Observatoire National sur la Formation, la Recherche et l'Innovation sur le Handicap. Rapport triennal ONFRIH - Synthèse et préconisation. http://www.ladocumentationfrancaise.fr/rapportspublics/114000304/index.shtml

[7] Jans, L.H., Scherer, M.J. (2006). Assistive technology training: Diverse audiences and multidisciplinary content. Disability and Rehabilitation: Assistive Technology, 1(1-2):

69-77. https://doi.org/10.1080/09638280500167290

[8] Fifield, M.G. Fifield, M.B. (1997). Education and training of individuals involved in delivery of assistive technology devices. Technology and Disability, 6(2): 7788. https://doi.org/10.3233/TAD-1997-61-207

[9] Rowley, B. A., Mitchell, D.F., Weber, C. (1997). Educating the rehabilitation engineer as a supervisor. Assistive Technology, 9: 62-69.

[10] Gray, T., Coleman, C. (1996). Rehabilitation engineering training program model and issues. Proceedings of the 1996 RESNA Annual Conference, RESNA, Washington, DC, pp. 5-7. 
[11] Winters, J.M. (1995). Rehabilitation engineering training for the future: Influence of trends in academics technology and health reform. Assistive Technology, 7: 95-110. https://doi.org/10.1080/10400435.1995.10132258

[12] Smith, R.O. (1992). Technology education from an occupational therapy view. Technology Disability, 1: 2230. https://doi.org/10.3233/TAD-1992-1306

[13] Candela, A.R. (2003). A pilot course in teaching skills for assistive technology specialists. Journal of Visual Impairment \& Blindness, 97(10): 661-666. https://doi.org/10.1177\%2F0145482X0309701012

[14] Lecomte, D. (2003). Aides techniques: Situation actuelle, données économiques, propositions de classification et de prise en charge. https://www.viepublique.fr/sites/default/files/rapport/pdf/034000170.pd $\mathrm{f}$

[15] Biard, N. (2019). L'apprentissage de procédures médicales par vidéo: effets de la segmentation et du contrôle du rythme par l'apprenant. https://tel.archivesouvertes.fr/tel-02020680/document

[16] Means, B., Toyama, Y., Murphy, R., Bakia, M., Jones, K. (2009). Evaluation of evidence-based practices in online learning: A meta-analysis and review of online learning studies. https://repository.alt.ac.uk/629/

[17] Arthanat, S., Elsaesser, L. and Bauer, S. (2017). A survey of assistive technology service providers in the USA. Disability and Rehabilitation: Assistive Technology, 12(8): 789-800. https://doi.org/10.1080/17483107.2016.1265015

[18] Somerville, N.J., Wilson, D.J., Shanfield, K.J., Mack, W. (1990). A survey of the assistive technology training needs of occupational therapists. Assistive Technology,

2(2): 41-49. https://doi.org/10.1080/10400435.1990.10132149

[19] Parkin, C., Hatzidimitriadou, E., Manship, S., Stein, M., Achille-Faveau, S., Blot, J., Laval, D. (2019). A survey of assistive technology (AT) knowledge and experiences of healthcare professionals in the UK and France: challenges and opportunities for workforce development. In: Special Thematic Session 15 Robotics and Virtual Worlds. https://kar.kent.ac.uk/76434/

[20] Hatzidimitriadou, E., Stein, M., Parkin, C., Manship, S., Gallien, P., Laval, D. (2019). Training needs and development of online AT training for healthcare professionals in UK and France. Association for the Advancement of Assistive Technology in Europe (AAATE), Bologne, pp. 27-30. https://kar.kent.ac.uk/76436/

[21] Leblong, E., Fraudet, B., Devigne, L., Babel, M., Pasteau, F., Benoit, N., Galien, P. (2020). SWADAPT1: Evaluation on standardised circuits of the interest of a robotic module for assisting the driver of an electric wheelchair: pilot, prospective, controlled, randomised study. In Annual Meeting and International Society of Physical \& Rehabilitation Medicine World Congress, ISPRM 2020.

[22] Cowan, D., Cudd, P., Judge, S. (2015). Over a decade of developing the assistive technology field in the UK. Technology and Disability, 27(1-2): 1-3. https://doi.org/10.3233/TAD-150428

[23] Clark, R.C., Nguyen, F., Sweller, J. (2006). Efficiency in Learning: Evidence-Based Guidelines to Manage Cognitive Load. John Wiley \& Sons. 